\title{
The role of microRNA-148a and downstream DLGAP1 on the molecular regulation and tumor progression on human glioblastoma
}

\author{
Yue $\mathrm{Li}^{1,2} \cdot$ Weiguo $\mathrm{Li}^{3}{ }^{3} \cdot$ Xiaomin Zeng $^{4} \cdot$ Xuemei Tang $^{1} \cdot$ Shan Zhang ${ }^{1} \cdot$ Feiya Zhong ${ }^{5} \cdot$ Xiangni Peng $^{6}$. \\ Yang Zhong ${ }^{6} \cdot$ Thomas J. Rosol $^{7} \cdot$ Xiyun Deng $^{1} \cdot$ Zhonghua Liu $^{8} \cdot$ Sihua Peng ${ }^{9,10,11} \cdot$ Xiaoning Peng $^{1,12}$
}

Received: 2 September 2018 / Revised: 24 April 2019 / Accepted: 26 May 2019 / Published online: 2 September 2019

(c) The Author(s) 2019. This article is published with open access

\begin{abstract}
Glioblastoma (GBM) is the most common malignant primary brain tumor in adults. Currently, the prognosis of the patients with GBM is very poor and new molecular targets and treatment strategies are urgently needed to combat it. MicroRNA148a (miR-148a) has been shown to be dysregulated in certain tumor types. However, the role of miR-148a in the pathogenesis of GBM is not fully understood. Here we comprehensively analyzed the roles of miR-148a, downstream DLGAP1, and their molecular pathways in GBM. We showed that miR-148a promote the proliferation and growth of GBM cells both in vitro and in vivo, and also induced the migration, invasion, and EMT (epithelial-mesenchymal transition) program of GBM cells by directly targeting DLGAP1. Furthermore, we identified 31 new miR-148a targets and found that miR-148a function was mainly involved in the cell adhesion signaling pathway and was associated with nervous system diseases. Our findings provide a new mechanism for miR-148a-mediated GBM cell invasion and reveal previously unreported targets of miR-148a as well as novel miR-148a-mediated regulatory networks in GBM. These results increase the understanding of the role of miR-148a in GBM and may lead to novel therapeutic strategies for GBM.
\end{abstract}

\section{Introduction}

These author contributed equally: Yue $\mathrm{Li}$, Weiguo $\mathrm{Li}$

Supplementary information The online version of this article (https:// doi.org/10.1038/s41388-019-0922-3) contains supplementary material, which is available to authorized users.

$\triangle$ Zhonghua Liu

liuzh@hunnu.edu.cn

$\triangle$ Sihua Peng

shpeng@ shou.edu.cn

$\triangle$ Xiaoning Peng

pxiaoning@hunnu.edu.cn

1 Department of Pathology and Pathophysiology, Hunan Normal University School of Medicine, Changsha 410013, China

2 Department of Pathology, Xiangtan Central Hospital, Xiangtan 411100, China

3 Department of Statistics, College of Mathematics and Computer Science, Hunan Normal University, Changsha 410081, China

4 Department of Epidemiology and Health Statistics, Public Health School, Central South University, Changsha 410078, China

5 Department of Genetics, College of Life Science, Wuhan University, Wuhan 430072, China
Glioma is the most common intracranial tumor, accounting for $70 \%$ of brain tumors [1]. According to the histopathological characteristics, the primary gliomas are divided into

6 Department of Information and Computing Science, School of Mathematics and Computational Science, Xiangtan University, Xiangtan 411105, China

7 Department of Biomedical Sciences, Heritage College of Osteopathic Medicine, Ohio University, Athens, OH 45701, USA

8 Department of Biochemistry and Molecular Biology, College of Life Science, Hunan Normal University, Changsha 410081, China

9 Key Laboratory of Exploration and Utilization of Aquatic Genetic Resources (Shanghai Ocean University), Ministry of Education, Shanghai 201306, China

10 National Pathogen Collection Center for Aquatic Animals, Ministry of Agriculture, Shanghai 201306, China

11 International Research Center for Marine Biosciences at Shanghai Ocean University, Ministry of Science and Technology, Shanghai 201306, China

12 Department of Pathophysiology, Jishou University School of Medicine, Jishou 416000, China 
four grades by the World Health Orgnization (WHO) [2]. WHO grade IV astrocytoma, known as glioblastoma (GBM), is characterized by high degree of malignancy, rapid progression, and marked invasion of the brain. GBM accounts for $3-4 \%$ of all cancer-related deaths [3]. Current treatment for GBM includes tumor resection with radiotherapy and chemotherapy; however, GBM has a very poor prognosis with a 5-year survival rate of $4-5 \%$ and a median survival of only 12.6 months $[3,4]$. The main causes of poor prognosis in patients with GBM include rapid cell growth, resistance to apoptosis, and invasion of the surrounding tissue $[2,5]$. The precise molecular mechanisms involved in GBM remain unclear.

A better understanding of the genetic and epigenetic events in GBM is necessary to identify the molecular mechanisms involved in GBM and novel molecular strategies to target GBM cells. The Cancer Genome Atlas (TCGA) project aims to perform a comprehensive analysis of the molecular characteristics of cancer patients using large sample data, which provides a basis for molecular classification and targeted therapy of cancers. GBM is the first tumor type to be analyzed by TCGA [6]. By mining the TCGA database, we identified a 17-RNA integrated signature consisted by 4 protective mRNAs, 12 risk-assoicated mRNAs, and 1 risk-associated microRNA (miRNA) that can predict the survival outcome of GBM patients more accurately than previously developed mRNA or miRNA signatures. We found a risk-associated miRNA, $h s a-m i R-148 a$, and a protective mRNA, DLGAP1 (human discs large homolog- associated protein 1), were upregulated and downregulated in GBM, respectively. The negative correlation between hsa-miR-148a and DLGAP1 was associated with survival of GBM patients. Interestingly, we also found that DLGAPl was one of the potential target genes of hsa-miR-148a by predictive bioinformatics [7]. Therefore, we suspect that miR-148a is involved in the progression of GBM by regulating DLGAP1.

Previous studies have shown that miR-148a was downregulated in many malignant tumors such as gastric cancer, liver cancer, pancreatic cancer, etc., and miR-148a suppressed tumor cell proliferation, angiogenesis, invasion, and metastasis [8-11]. These data suggest that miR-148a is a tumor suppressor gene in these tumors. Recently, it was found that the expression of miR-148a was upregulated in GBM and the high expression of miR-148a promoted the proliferation, migration, and invasion, and inhibited the apoptosis of GBM cells [5, 12, 13]. These findings indicate that miR-148a may have tissue-specific biological effects.

DLGAP1, also known as GKAP, SAPAP1, or DAP-1, belongs to the SAPAP family and is a postsynaptic protein, which plays an important role in the formation of synapses and the signaling of nerve cells $[14,15]$. The interactions between DLGAP1 and DLG-1 controls microtubule dynamics and promotes centrosome positioning in astrocytes. Centrosome positioning plays a very important role in cell division, differentiation, polarization, and migration [16]. Loss of the cell polarity can lead to tumorigenesis and metastasis [17-19]. Therefore, the role of miR-148a on the migration and invasion of GBM cells by regulating DLGAP1 is worthy of investigation.

miRNAs typically have hundreds of targets. At present, only several targets, such as MIG6 and BIM, were confirmed for miR-148a in GBM $[5,20]$. As miRNAs exert their effects in biologic networks, it is necessary to identify miR-148a targets in a global manner. A number of miRNA target prediction tools have been developed; however, their overlap in prediction is limited and there are inevitably false positives. Gene chip technology is often used to identify miRNA target genes but can only detect known genes and does not identify novel genes. This is not ideal for detecting low abundance mRNAs. As some of the target genes regulated by miRNA occurs at the translational level, mRNA expression can only partially explain miRNA target protein abundance. In recent years, proteomic methods were widely used to identify miRNA targets. Isobaric tags for relative and absolute quantification (iTRAQ) technology is an in vitro labeling technique for peptides. The technique uses four or eight isotope labels with specific labeling of the amino groups of the peptides, followed by tandem mass spectrometry (MS/MS) to compare the relative content of the proteins in the four or eight different samples. iTRAQ is characterized by high throughput, good repeatability, and high sensitivity [21].

In this study, we demonstrated that miR-148a was upregulated, DLGAP1 was downregulated in GBM, and DLGAP1 was a direct target of miR-148a. miR-148a promoted the proliferation and survival of GBM cells, and miR-148a promoted the migration, invasion, and EMT (epithelial-mesenchymal transition) of GBM cells, which was mediated by DLGAP1. We used iTRAQ technology combined with predictive bioinformatics tools, to identify the targets of miR-148a, and performed GO (Gene Ontology) functional enrichment analysis, GAD (Disease Associated Database) disease-enrichment analysis, and KEGG (Kyoto Encyclopedia of Genes and Genomes) pathway enrichment analysis for these targets. The results of this study discovered new targets of miR-148a and their molecular regulatory networks, which provided critical insight into the role of miR-148a in GBM.

\section{Results}

miR-148a was upregulated in glioma cell lines and patient tissues

To explore the role of miR-148a in GBM, we first detected the expression level of miR-148a in human astrocytes and 
A

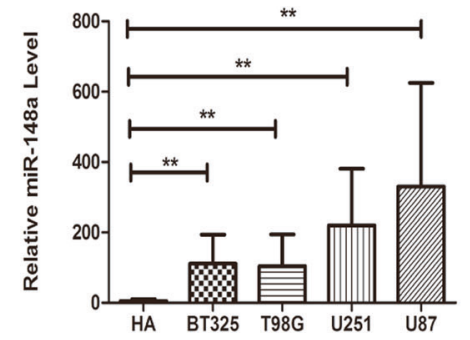

B

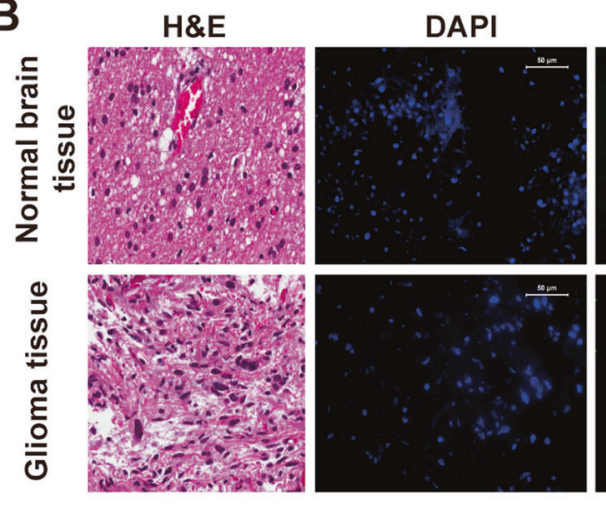

D
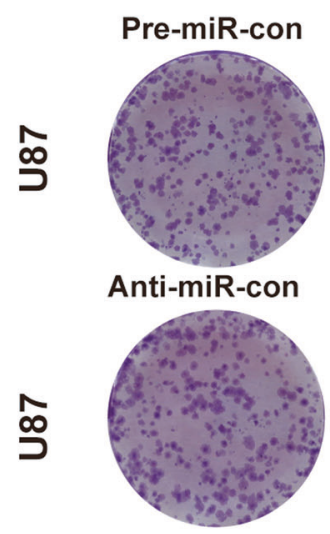

Pre-miR-148a

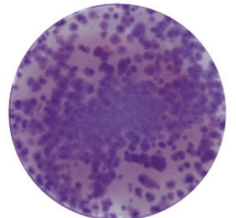

Anti-miR-148a

E

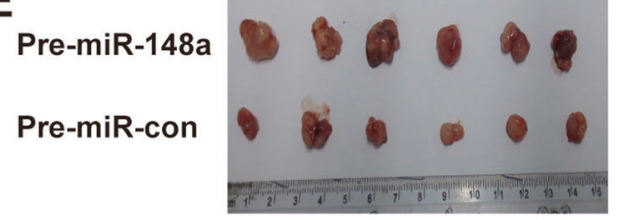

Anti-miR-148a

Anti-miR-con

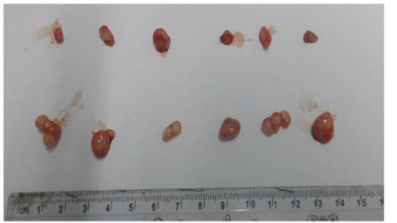

C
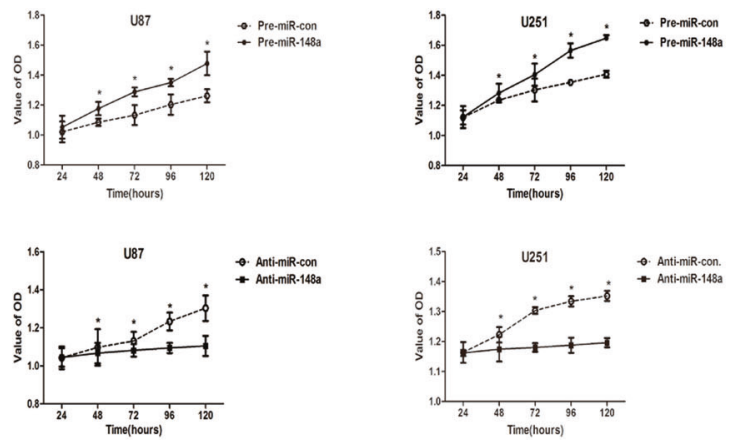

FITC
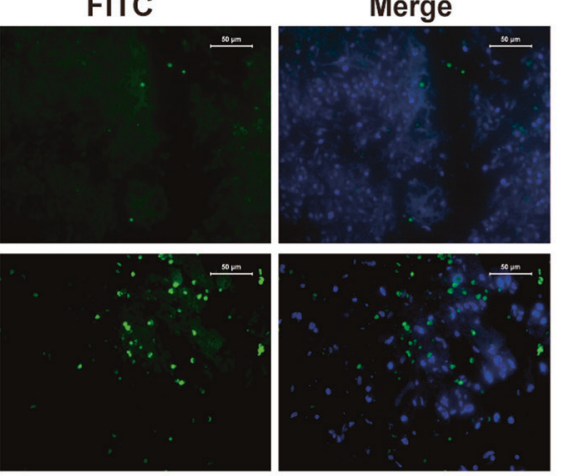

The sum of the fluorescence intensity

scores and the proportional scores.
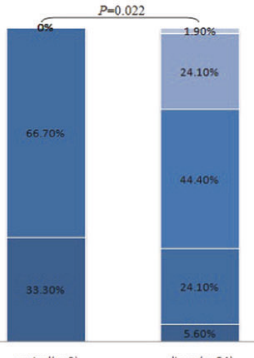

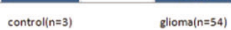

$\mathbf{F}$
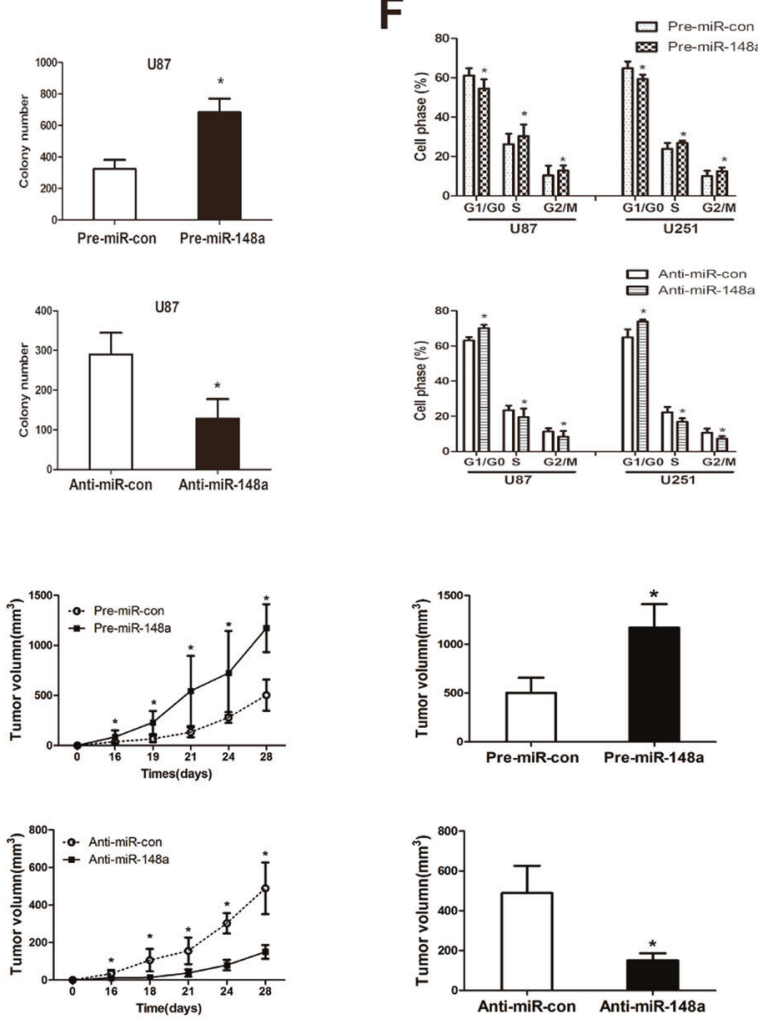

GBM cells, and showed that miR-148a was upregulated in GBM cells (BT325, T98G, U251, and U87) compared with human astrocytes (HA1800) (Fig. 1a). We then detected the expression level of miR-148a in a tissue array including 54 human gliomas and 3 normal brain tissues, and showed that the expression level of miR-148a in glioma tissues was higher than in normal brains $(P=0.022$; Fig. $1 b)$. We further analyzed the relationship between miR-148a and 
Fig. 1 The expression of miR-148a in GBM and effect of miR-148a on GBM cell proliferation. a MiR-148a was significantly upregulated in GBM cells (BT325, T98G, U251, and U87) compared with normal astrocyte (HA1800) $(* * P<0.01)$. b Representative image of $\mathrm{H} \& \mathrm{E}$ staining $(\times 40)$ and FISH staining of miR-148a (green) expression in a tissue array showed miR-148a localized in the cytoplasm. Scale bar, $50 \mu \mathrm{m}$. Statistical analysis indicated that miR-148a was upregulated in glioma tissues compared with normal human brain tissues $(* P<0.05)$. c CCK8 assay. The relative cell viability of the pre-miR-148a-treated groups of U87 and U251 cells was higher than pre-miR-con-treated groups, and the relative cell viability of the anti-miR-148a groups of U87 and U251 cells was lower than anti-miR-con-treated groups $(* P$ $<0.05$ ). d Colony-formation assay. Pre-miR-148a group had increased colony numbers compared with controls and the anti-miR-148a group had decreased the colony numbers compared with the anti-miR-con group $\left({ }^{*} P<0.05\right)$. e Tumor xenograft model. Tumor volumes and growth curves showed that tumors in the pre-miR-148a group grew more rapidly and the anti-miR-148a group grew more slowly $(* P<$ 0.05). $f$ Cell cycle analysis. Cells in $S$ and G2/M phase were increased by upregulating miR-148a and the cells in the $\mathrm{G}_{0} / \mathrm{G}_{1}$ phase of the cell cycle were increased by inhibiting miR-148a in both U87 and U251 cells $(* P<0.05)$. Error bars represent the mean \pm SD of three independent experiments

clinical and pathological features in patients with glioma and found that the expression of miR-148a was correlated with pathological grade $(P=0.004)$ but not with the age $(P=0.195)$ and sex of patients $(P=0.193)$ (Table 1$)$.

\section{miR-148a promoted the proliferation of GBM cells and enhanced tumor growth}

To explore the biologic significance of miR-148a in GBM, we established miR-148a- and anti-miR-148a-stable expressing GBM cells (with U87, U251 T98G, and BT325) using lentiviruses expressing miR-148a and anti-miR-148a, respectively. The efficacy of infection was detected by fluorescence microscopy and quantitative reverse transcriptase-PCR (qRT-PCR) (Supplementary Fig. S1). We examined the effects of miR-148a on cell proliferation using CCK8 and colony-formation assays. The results showed that miR-148a overexpression promoted GBM cell proliferation and the inhibition of miR-148a expression reduced the proliferation of GBM cells (Fig. 1c, d).

We used athymic nude mice with U87 flank tumors with pre-miR-148a or pre-miR-con, and anti-miR-148a or antimiR-con, to measure the effect of miR-148a on tumor growth in vivo. After inoculation for 16 days, tumor masses were present in 27 nude mice, whereas no tumors were present in only 1 nude mice in the anti-miR-148a group. This resulted in an overall tumorigenesis rate of $96.42 \%$ (27/28). Histopathology of flank xenografts is shown in Supplementary Fig. S2. Six nude mice in each group were included in the statistical analysis. On days 22 and 23 after inoculation, one mice died in the pre-miR-148a and pre-
Table 1 Relationship between miR-148a expression and clinicopathological parameters of glioma patients

\begin{tabular}{|c|c|c|c|c|c|c|}
\hline & \multicolumn{2}{|c|}{ Low } & \multicolumn{2}{|c|}{ High } & \multirow[t]{2}{*}{ Statistic } & \multirow[t]{2}{*}{$P$-value } \\
\hline & $n$ & $\%$ & $n$ & $\%$ & & \\
\hline Total $(n=54)$ & 16 & 29.6 & 38 & 70.4 & & \\
\hline Age (year) $(\bar{X} \pm S D)$ & \multicolumn{2}{|c|}{$50.8 \pm 10.5$} & \multicolumn{2}{|c|}{$55.3 \pm 12.0$} & $t=-1.313$ & 0.195 \\
\hline \multicolumn{7}{|l|}{ Gender $\left(n_{\text {missed }}=3\right)$} \\
\hline Male & 10 & 40.0 & 15 & 60.0 & $\chi^{2}=1.695$ & 0.193 \\
\hline Female & 6 & 23.1 & 20 & 76.9 & & \\
\hline \multicolumn{7}{|l|}{ Tumor stage } \\
\hline $\mathrm{I}+\mathrm{II}$ & 8 & 72.7 & 3 & 27.3 & $\chi^{2}=12.306$ & $<0.001$ \\
\hline III+IV & 8 & 18.6 & 35 & 81.4 & & \\
\hline I & 2 & 100.0 & 0 & 0.0 & $\chi^{2}=13.190$ & 0.004 \\
\hline II & 6 & 66.7 & 3 & 33.3 & & \\
\hline III & 2 & 20.0 & 8 & 80.0 & & \\
\hline IV & 6 & 18.2 & 27 & 81.8 & & \\
\hline
\end{tabular}

Three of the 54 cases in the tissue microarray lack gender information. The expression level of miR-148a was determined by the total value of fluorescence intensity and proportion of positive cells. The fluorescence intensity scores were $0=$ negative, $1=$ weakly positive, and 2 $=$ strongly positive, and the proportional scores were $0=0 \%, 1=$ $1-10 \%$ positive cells or diffused distribution, $2=11-50 \%$ positive cells or focal distribution, and $3 \geq 50 \%$ positive cells or widely distribution. The sum of the fluorescence intensity scores and the proportional scores $\geq 3$ represented a high miR-148a expression

miR-con groups. On day 22, skin ulcers occurred in one xenograft tumor in anti-miR-con group. Tumor volume was significantly different between the pre-miR-con and the premiR-148a groups $(P=0.002)$, and between the anti-miRcon and anti-miR-148a groups $(P<0.001)$. There were significant differences between the five time points of 16 , $19,21,24$, and 28 days $(P<0.001)$ within groups (pre-miR148a group: $P=0.001$; pre-miR-con group: $P<0.001$; antimiR-148a group: $P<0.001$; and anti-miR-con group: $P=$ $0.001)$. On the 28th day after inoculation, the size of transplanted tumors in pre-miR-148a, pre-miR-con, antimiR-148a, and anti-miR-con groups were $1173.03 \pm$ $238.74 \mathrm{~mm}^{3}, \quad 503.04 \pm 155.41 \mathrm{~mm}^{3}, \quad 150.03 \pm 36.16 \mathrm{~mm}^{3}$, and $488.92 \pm 137.21 \mathrm{~mm}^{3}$, respectively (Fig. 1e, Supplementary Table S1, and Supplementary Table S2). The results demonstraded that an overexpression of miR-148a increased the growth of GBM xenografts and inhibition of miR-148a expression decreased the growth of GBM xenografts.

To explore the potential mechanisms of miR-148a on GBM growth, flow cytometry showd that miR-148a overexpression increased the percentage of cells in S and G2/M phase, and inhibition of miR-148a expression increased the percentage of cells in the $G_{0} / G_{1}$ phase (Fig. 1f, Supplementary Fig. S3). These findings demonstrated that miR148a regulated GBM cell proliferation by modulating the cell cycle. 


\section{miR-148a promoted the migration, invasion, and EMT of GBM cells}

As invasion of surrounding tissues is an important feature of GBM, we analyzed the effects of miR-148a on the migration and invasion of GBM cells by scratch assay, transwell migration, and invasion assay. The results showed that the inhibition of miR-148a expression inhibited the migration and invasion of GBM cells, and overexpression of miR-148a promoted the migration and invasion of GBM cells (Fig. 2a, b). It is known that EMT plays an important role in the invasion of human GBM cells [22]. Therefore, we investigated whether miR-148a regulated EMT in GBM cells and increased the migration and invasion of GBM cells. By measuring the expression of EMT markers in GBM cells, we found that mesenchymal markers, N-cadherin, vimentin, MMP2, and MMP9, were expressed in U87, U251, BT325, and T98G cells, and epithelial marker E-cadherin was expressed only in the U251 cells (Fig. 2c). We found that overexpression of miR-148a inhibited the expression of Ecadherin and increased the expression of $\mathrm{N}$-cadherin, vimentin, MMP2, and MMP9 (Fig. 2d). These data demonstrated that high expression of miR-148a induced the migration, invasion, and EMT program of GBM cells.

\section{miR-148a targeted inhibition of DLGAP1 expression and was negatively correlated with DLGAP1 in glioma}

To clarify the mechanisms by which miR-148a induced EMT and invasion of GBM cells, it was necessary to identify miR148a targets. We previously found in GBM TCGA data mining that DLGAP1, a regulator of cell polarity and migration, was a potential target of hsa-miR-148a. We investigated whether miR-148a induced the invasion of GBM cells by regulation of DLGAP1. By detecting the expression levels of miR-148a and DLGAP1 in GBM, we found that DLGAP1 mRNA and protein were downregulated in GBM cells (U251, U87, BT325, and T98G) compared with human astrocytes (HA1800) (Fig. 3a, b). We also found that the expression of DLGAP1 in glioma tissues was lower than that in normal brain $(P=0.010$, Fig. $3 c)$ and was not correlated with pathological tumor grade $(P=0.279$, Supplementary Table S3). miR-148a was negatively correlated with DLGAP1 in glioma tissues $\left(r_{\mathrm{s}}=-0.541, P<0.001\right)$ (Supplementary Table S4). To confirm whether DLGAP1 is a direct target of miR-148a, we constructed wild-type and mutant DLGAP1 3'-untranslated region (UTR) and cloned them downstream of the luciferase gene. Dual luciferase reporter assay showed that overexpression of miR-148a significantly inhibited the luciferase activity with wild-type DLGAP1 3'-UTR but not the mutant DLGAP1 3'-UTR (Fig. $3 \mathrm{~d})$. We also found that overexpression of miR-148a inhibited endogenous DLGAP1 and inhibition of miR-148a promoted the expression of endogenous DLGAP1 mRNA and protein levels (Fig. 3e, f). These results demonstrated that miR-148a inhibited the expression of DLGAP1 by directly binding to the DLGAP1 3'-UTR terminus.

\section{DLGAP1 mediated the effect of miR-148a on EMT program in GBM}

To investigate whether DLGAP1 mediates the role of EMT in GBM, we transfected the shRNA-DLGAP1 virus into U87 and U251 GBM cells. The efficacy of infection was detected by fluorescence microscopy and qRT-PCR (Supplementary Figure S4). Downregulation of DLGAP1 decreased Ecadherin in shRNA-DLGAP1-U251 cells and increased Ncadherin, vimentin, MMP2, and MMP9 in shRNA-DLGAP1U251 and shRNA-DLGAP1-U87 cells (Fig. 4a). Downregulation of DLGAP1 increased the ability of GBM cells to migrate and invade (Fig. 4b, c). These results demonstrated that DLGAP1 increased EMT program in GBM.

To investigate whether DLGAP1 mediated the effect of miR-148a on EMT in GBM, we transfected the shRNADLGAP1 virus into anti-miR-148a-U87 and anti-miR-148aU251 cells, and measured the expression levels of EMT protein markers. N-cadherin, vimentin, MMP2, and MMP9 were increased in anti-miR-148a-U87 and anti-miR-148a-U251 cells; however, E-cadherin was decreased in anti-miR-148aU251 cells (Fig. 4d). We found that the inhibition of DLGAP1 partially reversed the effect of miR-148a inhibition on the migration and invasion of GBM cells (Fig. 4e, f). In summary, miR-148a promoted the migration, invasion, and EMT program of GBM cells, which was mediated by DLGAP1.

\section{The effect of miR-148 on the glioma cell proteome}

It is generally estimated that each miRNA has hundreds of targets. The interaction between miR-148a and DLGAP1 cannot completely explain or address the biological phenotype of miR-148a. Therefore, we used the iTRAQ technique to analyze the protein expression profiles of anti-miR148a-U87 and anti-miR-con-U87 cells. To maximize the accuracy, 2 biological replicates were used in each group and a total of 4329 proteins were identified. We first standardized the ratio of protein expression of anti-miR-148U87 to anti-miR-con-U87 cells by $\log 2$ and then conducted differential protein screening based on a differential expression threshold of mean $\pm 1.96 \mathrm{SD}$. Eighty upregulated proteins and 136 downregulated proteins were obtained based on the threshold of 117:115 $(0.99,-1.06), 80$ upregulated proteins and 151 downregulated proteins on the 117:116 (0.91, -0.98), 102 upregulated proteins and 90 downregulated proteins on the 118:115 $(0.8,-0.8)$, and 108 upregulated proteins and 102 downregulated proteins on the 
A Anti-miR-con Anti-miR-148a

Oh

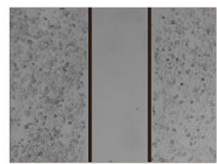

$4 \mathrm{~h}$

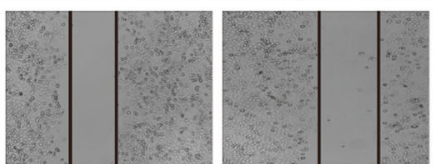

U87

Pre-miR-con Pre-miR-148a

Oh
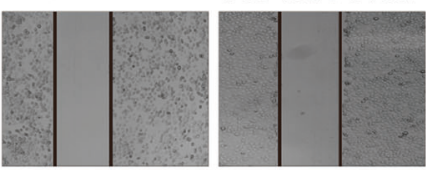

$24 \mathrm{~h}$

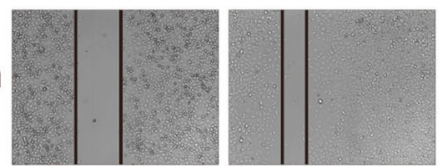

บ87
Anti-miR-con Anti-miR-148a
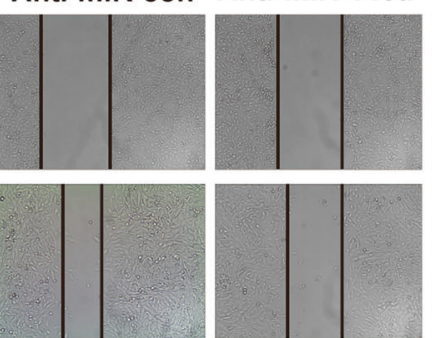

U251

Pre-miR-con Pre-miR-148a
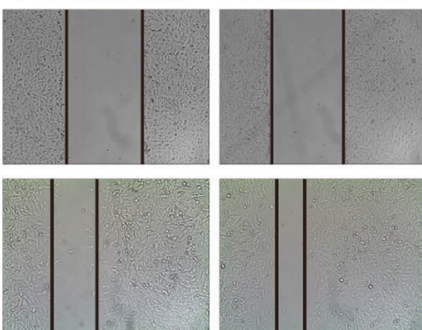

U251

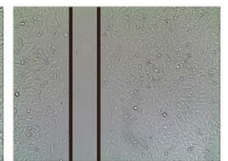

Anti-miR-con Anti-miR-148a

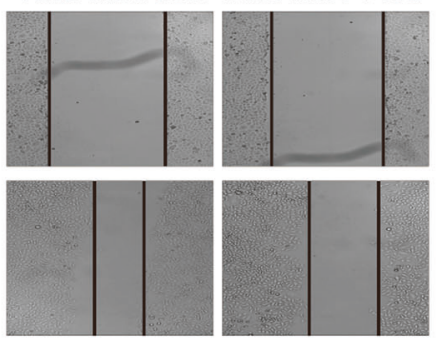

BT325
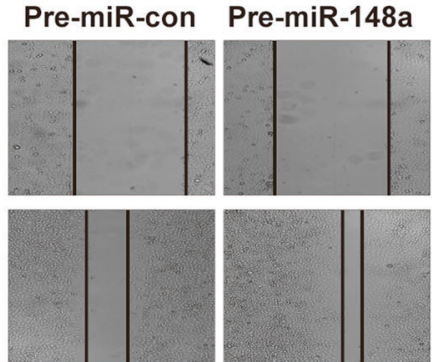

BT325

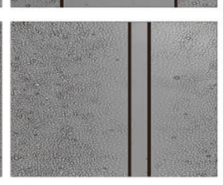

B

U87

Anti-miR-con Anti-miR-148a
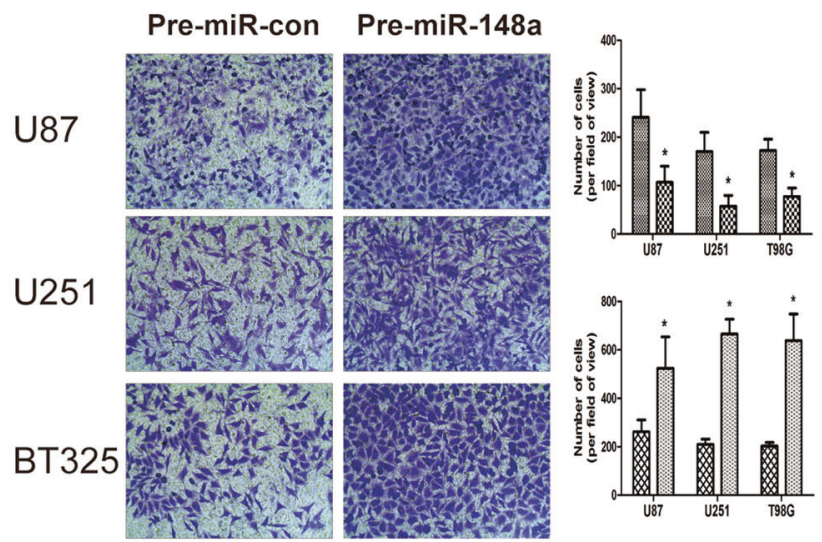

AntimiR-con

U251
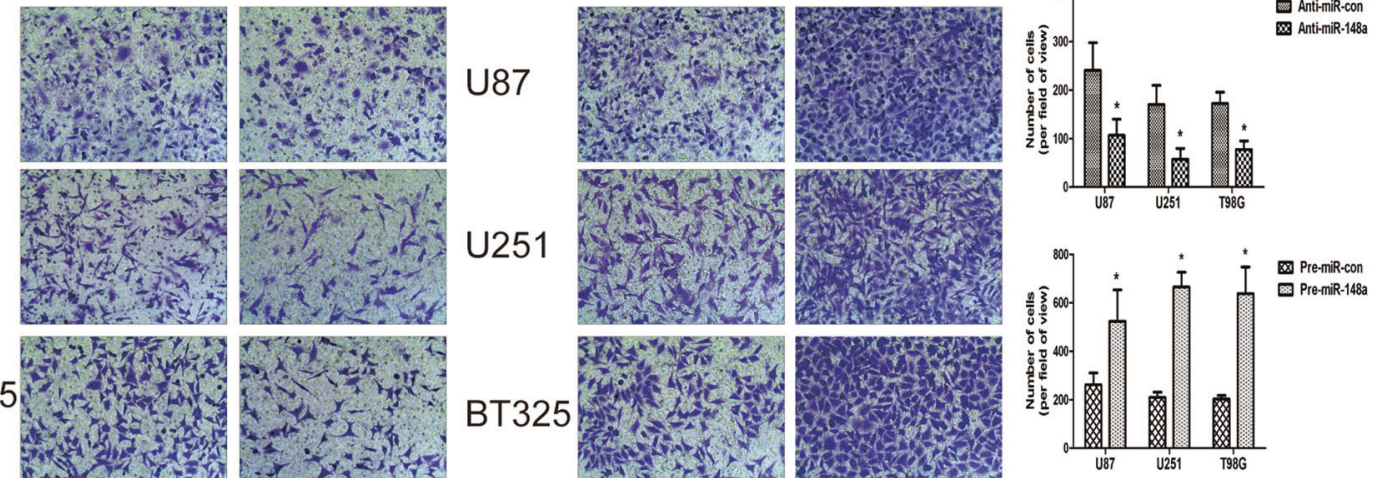

C

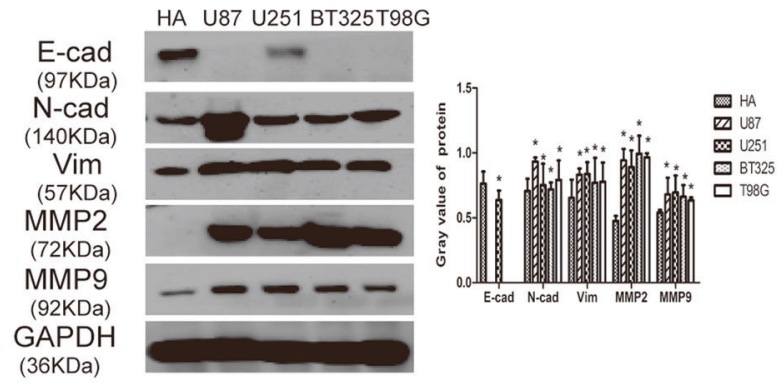

Fig. 2 The effect of miR-148a on the migration, invasion, and EMT of GBM cells. a Wound-healing assay. The inhibition of miR-148a expression impaired the migration of GBM cells and overexpression of miR-148a promoted the migration of GBM cells. b Tranwell assay. The inhibition of miR-148a expression impaired the invasion of GBM cells and overexpression of miR-148a promoted the invasion of GBM cells. The panels show the number of cells in transwell experiment $(* P<0.05)$. c Western blotting analysis of the expression of mesenchymal markers in GBM cells. N-cadherin, vimentin, MMP2,

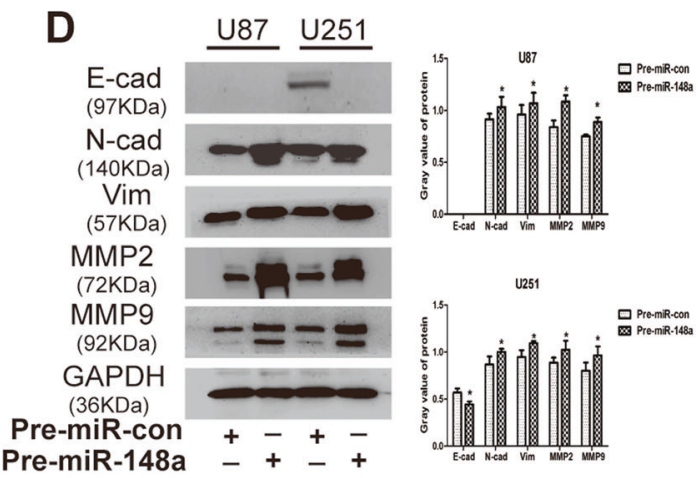

and MMP9 were expressed in U87, U251, BT325, and T98G cells and the normal astrocytes (HA1800), but epithelial marker E-cadherin was only expressed in U251 cells and normaol astrocytes (HA1800). The panels show the value of preoteins $(* P<0.05)$. d Overexpression of miR-148a inhibited the expression of E-cadherin and promoted the expression of N-cadherin, vimentin, MMP2, and MMP9. GAPDH was used as a control. The panels show the value of preoteins $(* P<0.05)$. Error bars represent the mean $\pm \mathrm{SD}$ of three independent experiments 
A

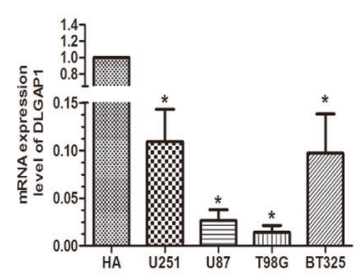

C

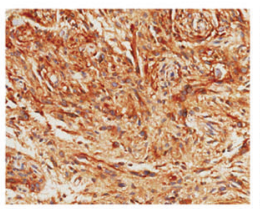

Normal brain tissue

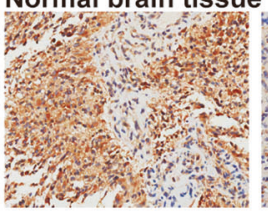

Grade III

E
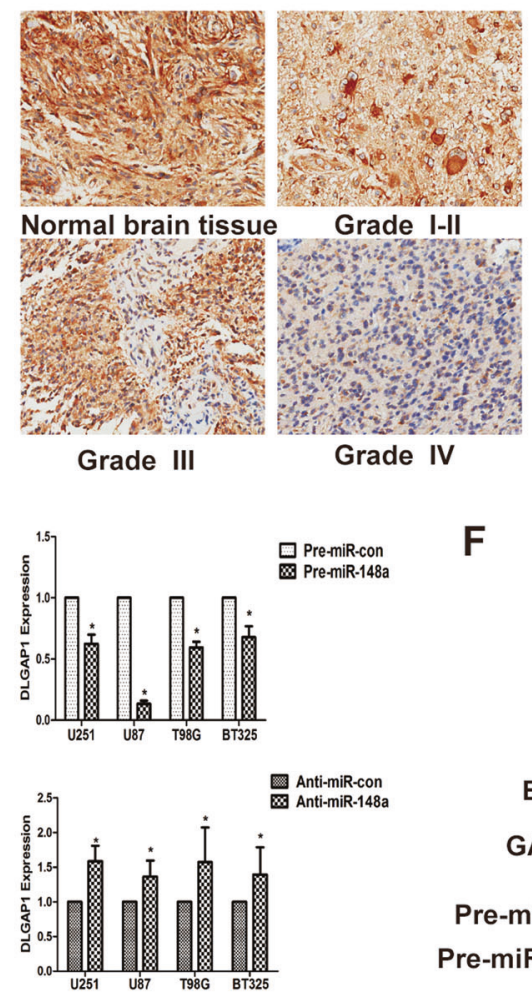

F
B
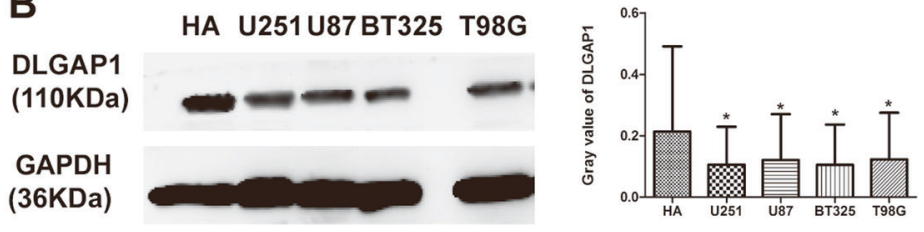

D

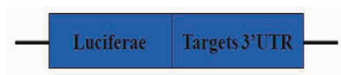

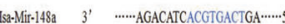

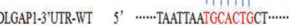

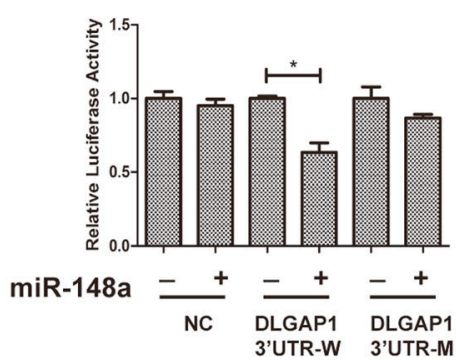

miR-148a
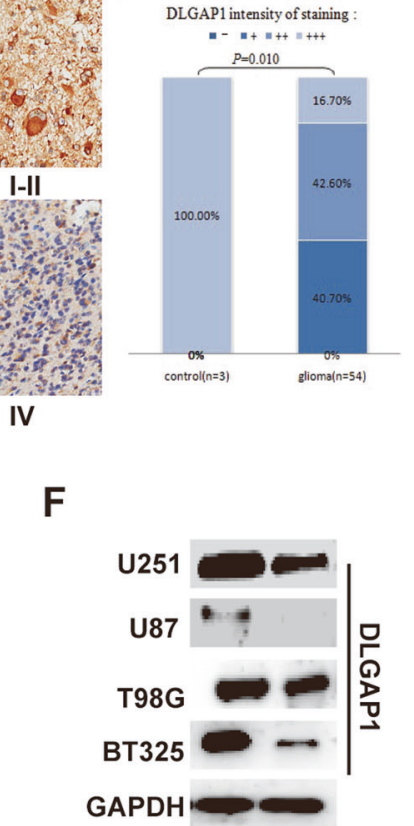

Pre-miR-con + -

Pre-miR-148a - +

Fig. 3 DLGAP1 was a direct target gene of miR-148a. a qRT-PCR analysis of DLGAP1 mRNA expression in normal astrocytes (HA1800) and GBM cells (U251, U87, T98G, and BT325) $(* P<$ $0.05)$. b Western blotting analysis of DLGAP1 protein expression in normal astrocytes (HA1800) and GBM cells (U251, U87, BT325, and T98G) $(* P<0.05)$. c Representative images of DLGAP1 immunohistochemistry in normal brain and glioma tissues. Statistical analysis indicated that DLAGP1 was downregulated in glioma tissues compared with that in human normal brain tissues $(* P<0.05)$. d Schematics of predicted binding sites of miR-148a in the wild-type
DLGAP1-3'-UTR and mutated sequence of DLGAP1-3'-UTR. Dual luciferase reporter assay showed that overexpression of miR-148a could significantly inhibit the luciferase activity of wild-type DLGAP1 3'-UTR, but unaffected the luciferase activity of mutant DLGAP1 3'UTR $(* P<0.05)$. e, f Overexpression of miR-148a inhibited the expression of endogenous DLGAP1 and the inhibition of miR-148a promoted the expression of endogenous DLGAP1 mRNA and protein, as determined by qRT-PCR and western blotting $(* P<0.05)$. Error bars represent the mean $\pm \mathrm{SD}$ of three independent experiments
118:116 (0.74, -0.76) (Fig. 5a, d). There were 224 proteins that had the same upregulated or downregulated expression trends. Therefore, the 224 proteins were considered to be regulated by miR-148a, including 78 upregulated proteins and 146 downregulated proteins (Supplementary Table S5).

\section{Functional enrichment of miR-148a-regulated proteins}

To further understand the role of miR-148a, DAVID online software was employed to analyze the $224 \mathrm{miR}-148 \mathrm{a}$ regulated proteins [23]. Using GO function analysis, we found that the most significant biological processes was cell-cell adhesion, which is associated with tumor invasion and metastasis [24] (Fig. 6a). The studies showed that miR148a-regulated proteins, such as GADD45A, GRP78, PARK7, and S100P, are invovled in cell-cell adhesion and are associated with the formation and invasion of glioma [25-28]. In addition, miR-148a also regulates other important biological processes, including apoptosis, DNA repair, mitosis cycle regulation, cell proliferation, nucleosome assembly, nucleocytoplasmic transport, and translational initiation, and these are related to the occurrence and development of cancer. Using GAD disease enrichment 
A

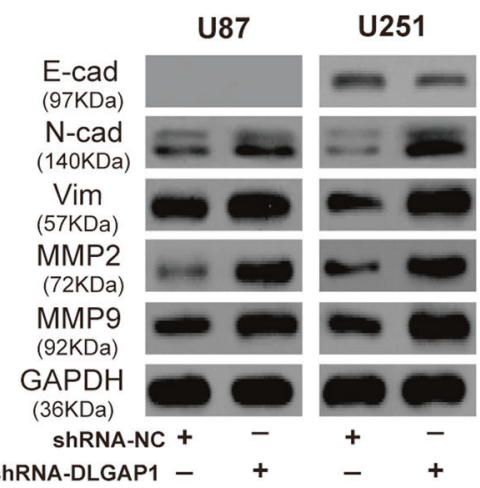

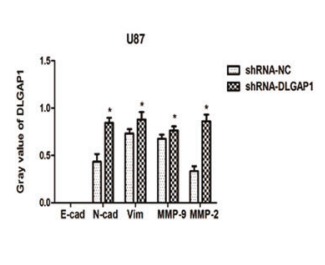

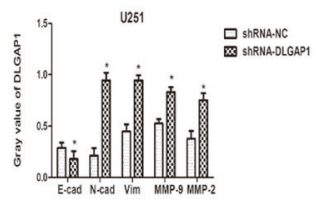

C
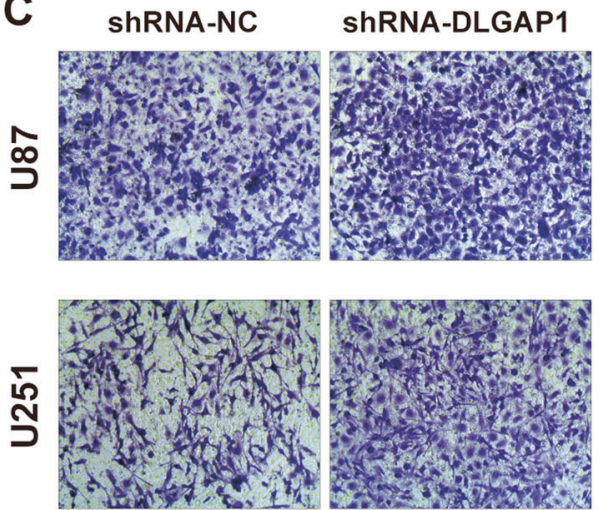

B

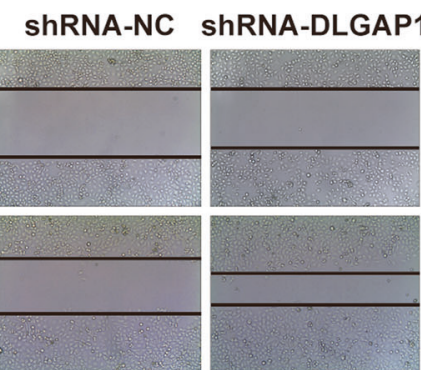

U87

D
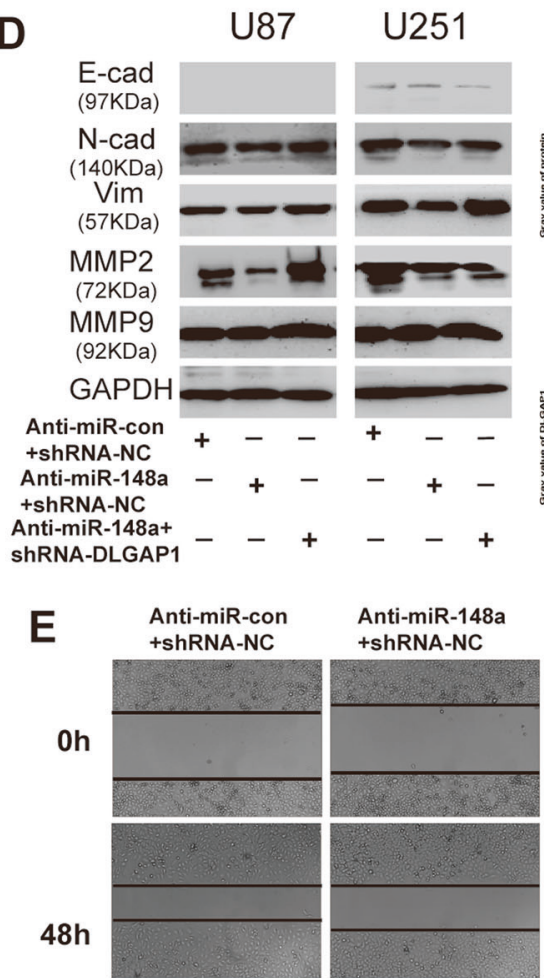

Anti-miR-148a +shRNA-NC

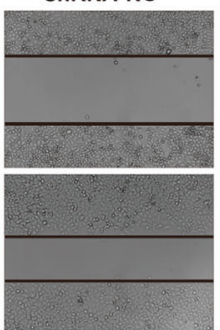

U87

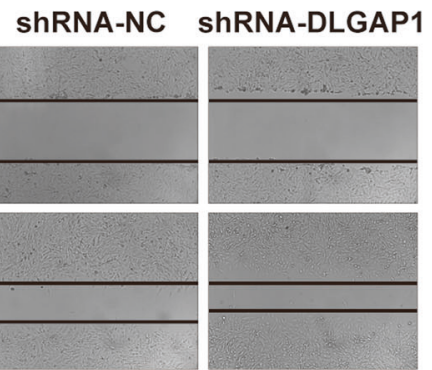

U251
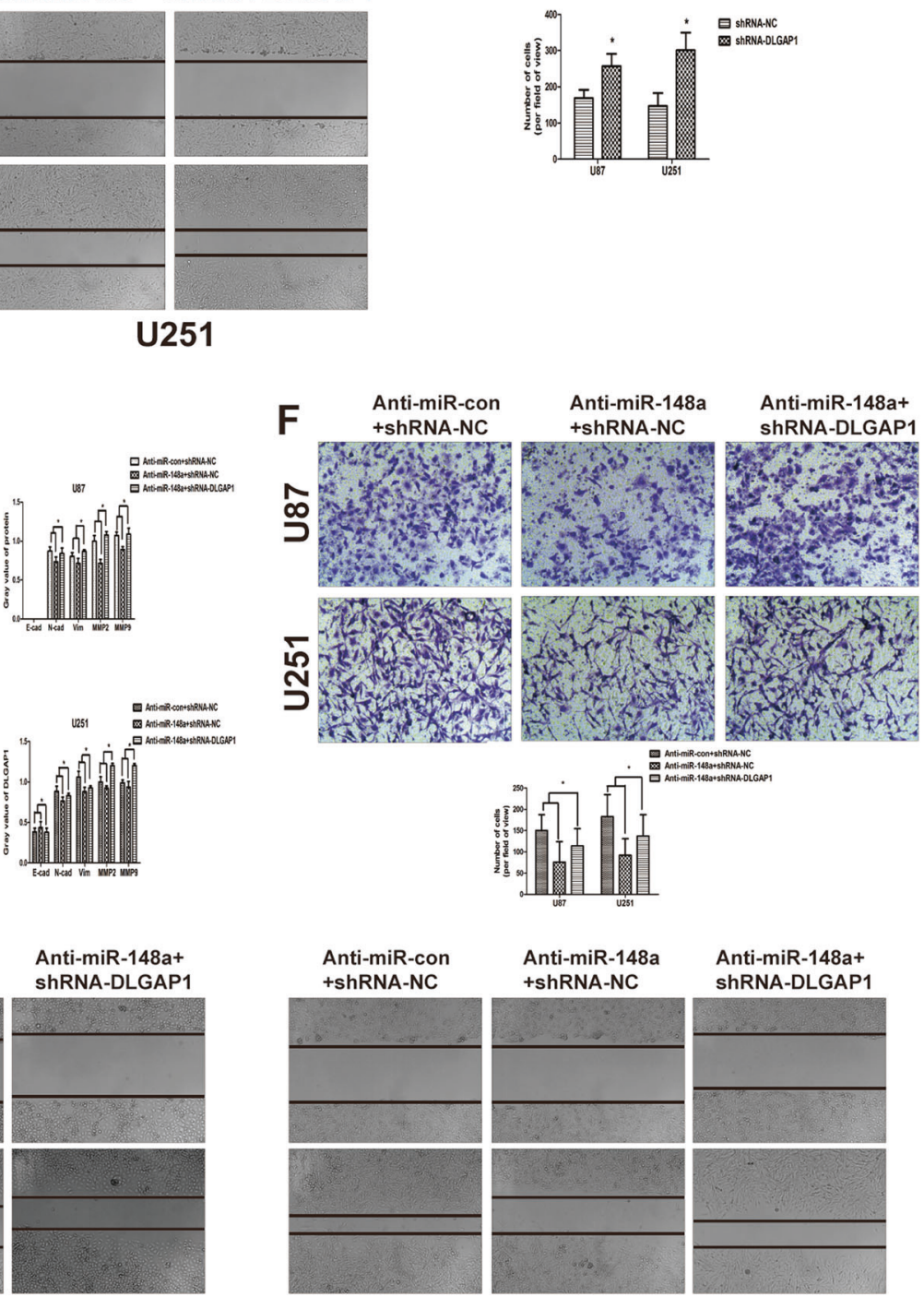

Anti-miR-148a+ ShRNA-DLGAP1

U251

analysis, we found that the most significant enrichment of disease was neurodegenerative or aging diseases, such as acognitive trait, aging/telomere length, brain ischemia, and
Parkinson's disease. Using KEGG pathway analysis, we found that the most significant pathway enrichment was related to Alzheimer's disease (Fig. 6b), which is consistent 
Fig. 4 DLGAP1 mediated the effect of miR-148a on EMT in GBM. a EMT-associated proteins were regulated by silencing DLGAP1: downregulation of E-cad and upregulation of N-cad, vim, MMP2, and MMP9 at the protein level $(* P<0.05)$. b Wound-healing assays showed the downregulation of DLGAP1 increased GBM cell migration. c Tranwell assay showed that silencing DLGAP1 induced the U87 and U251 cells invasion $(* P<0.05)$. d Co-transfection of antimiR-148a and shRNA-DLGAP1 partly restored the decrease in N-cad, vim, MMP2, and MMP9 mRNA caused by anti-miR-148a in both U87 and $\mathrm{U} 251$ cells, and the increase of E-cad caused by anti-miR-148a in U251 cells $(* P<0.05)$. e, f Wound-healing assays and Transwell assay. The inhibition of DLGAP1 partially reversed the effect of miR148a inhibition on the migration and invasion of GBM cells. $\left({ }^{*} P<\right.$ 0.05 ). Error bars represent the mean $\pm \mathrm{SD}$ of three independent experiments

with previous studies that miR-148a was associated with the development of the nervous system and neurodegenerative diseases [29].

\section{Analyses of miR-148a target genes}

To identify the target genes of miR-148a, the STarMirDB database [30] was used to analyze the binding sites of miR$148 \mathrm{a}$ at the 224 miR-148a-regulated genes. It was shown that 10 genes had miR-148a-binding sites in the $3^{\prime}$-UTR, 2 genes had miR-148a-binding sites in the $3^{\prime}$-UTR and $5^{\prime}$ UTR, 10 genes had miR-148a-binding sites in their $3^{\prime}$-UTR and CDS (Coding Sequence)), and 20 genes had miR-148abinding sites in their CDS. As experimental verification is an important step in identifying target genes of a miRNA, we used TarBase V8.0 [31], an experimentally confirmed miRNA target database, to confirm the 42 possible target genes. We found that 11 of the 42 (26\%) genes were previously identified as miR-148a targets by biological experiments. Four were upregulated and seven were downregulated, including PDIA3, MTPN, SNX4, and COX5a, which are related to the development of cancer. The other 31 genes have not been reported and are considered novel target genes of miR-148a. Sixteen were upregulated and 15 were downregulated, including CASP8, SKP1, and MBNL1, which were related to the development of glioma (Supplementary Table S6). To validate these novel miR-148a candidate targets, we employed dual luciferase reporter assays to examine the effect of miR-148a overexpression on several targets, including AAGAB, MBNL1, STX12, NASP, and SKP1. The results of dual luciferase reporter gene assay showed that overexpression of miR-148a significantly inhibited the luciferase activities with wild-type $3^{\prime}$-UTR of AAGAB, MBNL1, STX12, NASP, and SKP1, but not the mutant $3^{\prime}$-UTR of AAGAB, MBNL1, STX12, NASP, and SKP1 (Fig. 6c and Supplementary Figure S5). The results confirmed that they were the direct targets of miR-148a. Taking together, these evidences confirmed that these 42 genes obtained by
STarMirDB database analysis of binding sites between miR-148a and target genes are the target genes of miR$148 \mathrm{a}, 31$ of which are newly identified miR-148a target genes.

\section{miR-148a-regulated protein-protein network}

Based on these 42 miR-148a target genes discovered in this study, a protein-protein interaction (PPI) network regulated by miR-148a was constructed by using the protein interaction database HPRD9 [32]. The network consisted of 472 protein interactions and 436 nodes (Fig. 6d). By analyzing the degree of the network, we found that the target gene of miR-148a had more interacting proteins. The average degree of miR-148a target gene was 11.6, whereas the average degree of non-miR-148a target gene was 1.06. For example, the degrees of CASP8, RASA1, SKP1, PDIA3, SET, and SNX4 were 72, 66, 45, 13, 25, and 10 in the network, respectively, indicating that CASP8, RASA1, SKP1, PDIA3, SET, and SNX4 were hubs of this network (CASP8, RASA1, and SKP1 were new targets of miR-148) and were involved in apoptosis, cell cycle, DNA repair, cell redox homeostasis, and other tumor processes.

\section{Disscusion}

In a previous study, we found miR-148a was inversely correlated with GBM patient survival by interrogating a TCGA database [7]. Here we confirmed that the expression of miR-148a was significantly increased in GBM cells compared with human astrocytes and was correlated with the degree of malignancy in glioma.

We found that overexpression of miR-148a promoted the proliferation, growth, migration, and invasion of GBM cells. Conversely, inhibition of miR-148a expression inhibited the proliferation, growth, migration, and invasion of GBM cells. The biological function of miR-148a in GBM was also confirmed in a xenograft model in vivo. Our results are consistent with the recent results from Kim et al. [5]. We have demonstrated for the first time that miR-148a promoted the migration and invasion of GBM cells and induced EMT through DLGAP1, which is a new discovery on the role of miR-148a in GBM.

EMT is a process in which epithelial cells are transformed into mesenchymal cells and mediates cancer cell invasion [33, 34], which is an important step in tumor metastasis [35]. The pathological features of EMT include decreased epithelial marker E-cadherin and increased mesenchymal cell markers $\mathrm{N}$-cadherin, fibronectin, and vimentin, decreased cell adhesion, and increased motility [36]. Studies have shown that primary GBM and its stem cell lines have the molecular characteristics of mesenchymal 

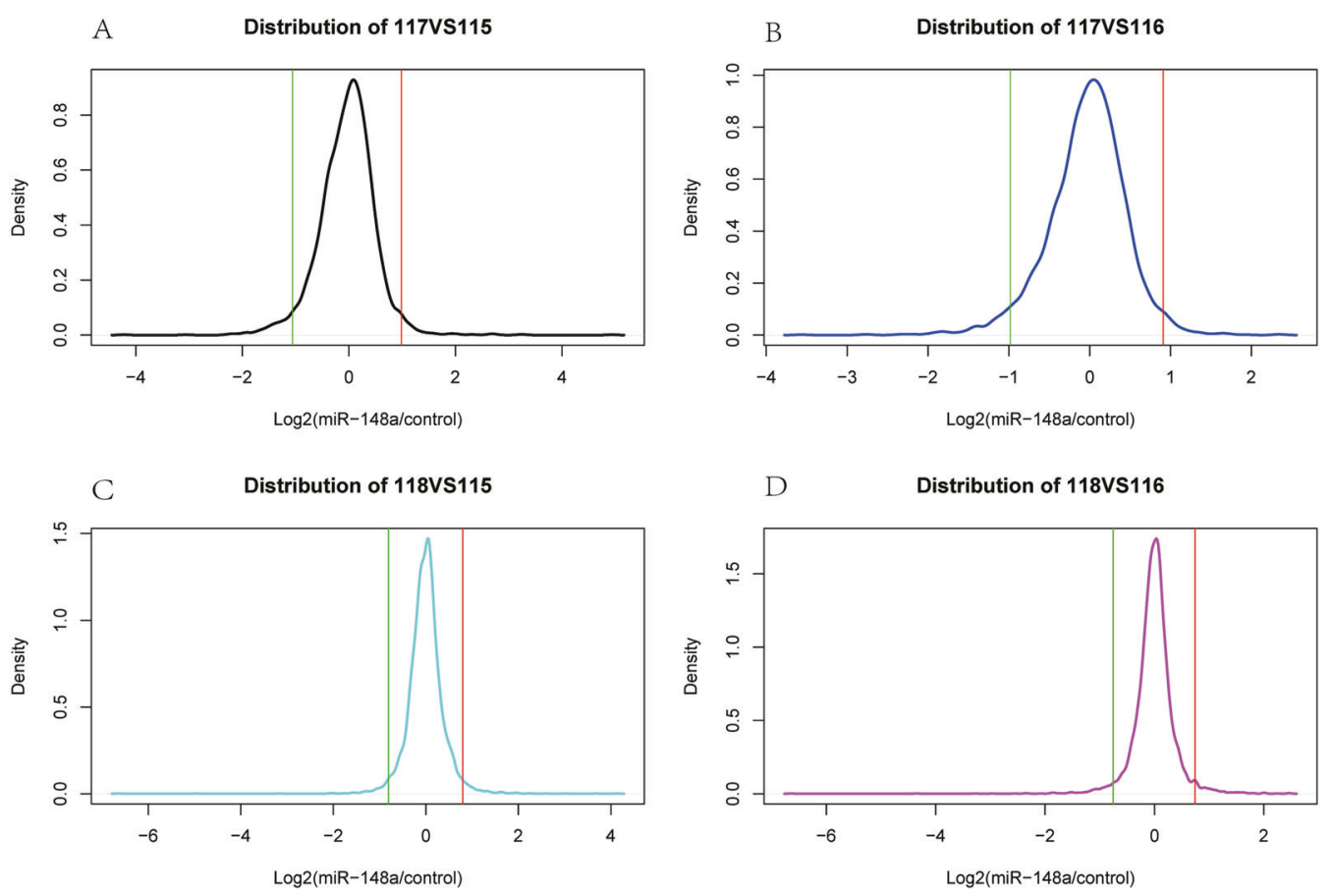

Fig. $5 \log 2$ protein expression ratios distribution of 4329 proteins of anti-miR-148a-U87 cells to anti-miR-con-U87 cells. The ratios of protein expression of anti-miR-148a-U87 cells to anti-miR-con-U87 cells were normalized by $\log 2$ and the differential expression thresholds were based on mean $\pm 1.96 \mathrm{SD}$, marked with vertical lines, which represent thresholds in protein upregulation or downregulation. a The protein expression ratio distribution of anti-miR-148a-U87 cells (117)

stem cells with the expression of mesenchymal-related genes, such as YKL-40, tenascin C, osteonectin, and CD105 [37]. Overexpression of TWIST, which can promote the occurrence of EMT, significantly enhanced the invasion of GBM cells [38]. In this study, we found mesenchymal markers $\mathrm{N}$-cadherin and vimentin were expressed in four GBM cell lines; however, E-cadherin was only expressed in the U251 GBM cells. We further confirmed that miR-148a inhibited the expression of E-cadherin in U251 GBM cells and promoted the expression of $\mathrm{N}$-cadherin, vimentin, MMP2, and MMP9 in proteins in U87 and U251 GBM cells. These findings demonstrated that EMT plays an important role in the survival and invasion of GBM cells and miR-148a promotes EMT in GBM cells.

Our previous data-mining studies have found that DLGAPI was downregulated in GBM and is one of the potential target genes of hsa-miR-148a [7]. In this study, we confirmed that DLGAPI was downregulated in GBM and was a direct target gene of miR-148a by luciferase reporter assay. DLGAP1 is polarity protein, which regulates cell development, maintenance of cell polarity, damage repair, and tissue integrity [39]. Changes in cell polarity may promote tumorigenesis, EMT, and cell invasion and metastasis [40]. To understand the effect of DLGAP1 on GBM cell migration and invasion or EMT, we knocked down DLGAP1 in GBM cells by shRNA-

to anti-miR-con-U87 cells (115). b The protein expression ratio distribution of anti-miR-148a-U87 cells (117) to anti-miR-con-U87 cells (116). c The protein expression ratio distribution of anti-miR-148aU87 cells (118) to anti-miR-con-U87 cells (115). d The protein expression ratio distribution of anti-miR-148a-U87 cells (118) to antimiR-con-U87 cells (116)

DLGAP1 and found that downregulation of DLGAP1 expression enhanced the ability of glioma cell migration and invasion, downregulated E-cadhern, and upregulated N-cadhern, vimentin, MMP2, and MMP9. We also knocked down DLGAP1 in anti-miR-148a GBM cells and found that knocking down DLGAP1 partially prevented the effects of miR-148a inhibition on cell migration and invasion, and EMT, suggesting that DLGAP1 mediated the effects of miR-148a.

It is naive to interpret the biological phenotype of a miRNA only through the interaction with a single target. Therefore, we identified the protein expression profiles regulated by miR148a by using iTRAQ in GBM. For the first time, the biological functions of miR-148a in GBM were revealed from a global perspective and new target genes of miR-148a were identified. By constructing a miR-148a-regulated protein interaction network, we further analyzed the molecular regulatory characteristics of miR-148a in GBM.

Through GO enrichment analysis, we found that miR148a-regulated proteins were significantly enriched in the cell-cell adhesion process, which is often associated with tumor invasion. For example, the enriched genes (GRP78, $P A R K 7$, and S100P) are related to the growth, metastasis, and invasion of glioma. GAD disease enrichment showed that miR-148a-regulated proteins were enriched in neurodegenerative or aging diseases, such as cognitive trait, 

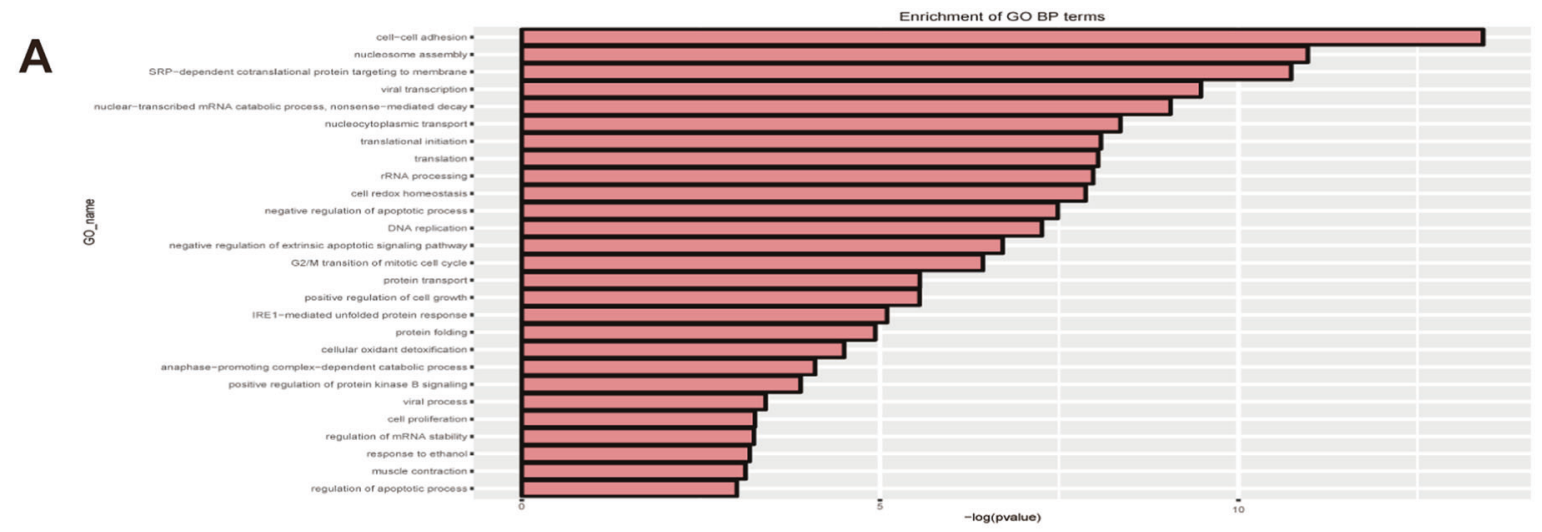

B

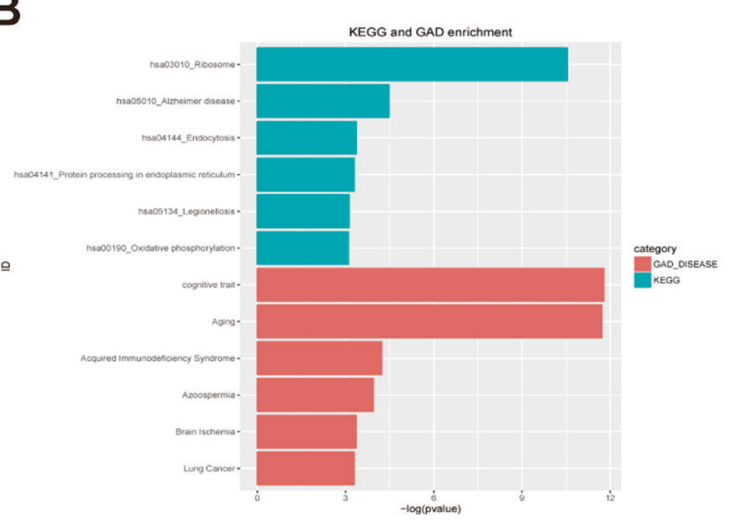

C
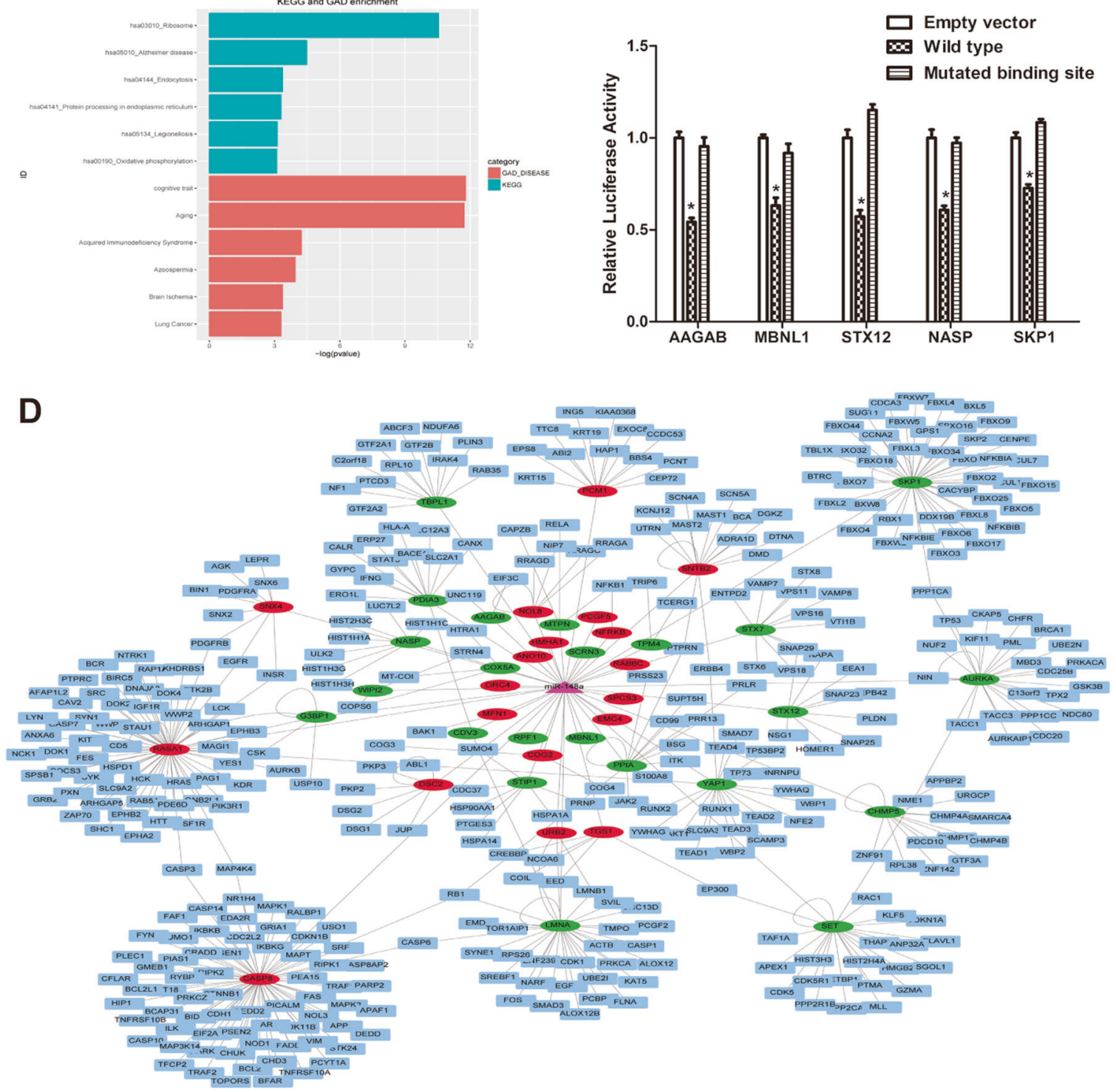

aging/telomere length, and brain ischemia. The KEGG pathway enrichment analysis showed that the miR-148aregulated proteins were significantly enriched in the
Alzheimer's disease pathway. Epidemiological studies showed that Alzheimer's disease can increase the risk of GBM and there is a similar gene expression pattern between 
Fig. 6 Biological function of the miR-148a-regulated proteins and PPI analysis of the miR-148a-targeted proteins. a GO biological processes enrichment analysis for the 224 miR-148a-regulated proteins. b KEGG and GAD enrichment analysis for the 224 miR-148a-regulated proteins. The statistical significance $P$-value was negative 10 -based $\log$ transformed. c Dual luciferase reporter assay to evaluate the effect of miR-148a overexpression on targets identified from proteomic experiments, including AAGAB, MBNL1, STX12, NASP, and SKP1 $(* P<0.05)$. d PPI network of the 42 miR-148a-targeted proteins. In the network, the triangle denotes the miR-148a, the red ellipses denote the upregulated targets, the green ellipses denote the downregulated targets, and the blue rounded rectangles denote genes connected with targets

Alzheimer's disease and GBM [41]. Therefore, we can infer that low expression of miR-148a leads to neurodegenerative diseases such as Parkinson's and Alzheimer's disease, and high expression of miR-148 may cause the abnormal proliferation of glial cells and leads to GBM.

By analyzing the target binding sites of miRNA-148a, 42 possible miR-148a direct targets were identified. Eleven were confirmed as targets of miR-148a by previous biological experiments and the other 31 represent newly identified targets of miR-148a, several of which were confirmed by luciferase-3'-UTR assays. These evidences together confirmed that these 42 genes were the direct targets of miR-148a. PPIs are crucial to biological processes, so studying the relationship between PPI networks and miRNA regulation will help to understand the influence of a miRNA on protein interactions and their cellular mechanisms. Previous studies have shown that miRNA targets are often hub nodes in the PPI network and have many important biological functions [42]. We found that the targets of miR-148a interacted with more proteins, were often the hub nodes in the network, and involved important molecular mechanisms of GBM. For example, CASP8 is a member of Caspase family. The common D302H variant of CASP8 and methylation of the CASP8 gene promoter are associated with the risk of glioma $[43,44]$. SKP1 is a scaffold protein involved in maintaining normal centromeric function [45]. It is also a component of ubiquitin ligase E3, which participates in the ubiquitination pathway to degrade cell cycle regulators (such as p21 and p27) [46]. Abnormal expression of SKP1 leads to genomic instability and tumor transformation [47]. It was interesting to identify RASA1 in the network. RASA1 is a tumor suppressor [48, 49] that interacts with EGFR proteins. The RASAl gene is amplified and overexpressed in about $40 \%$ of GBM patients [50]. This suggests miR-148a participates in GBM development through the EGFR (Epidermal Growth Factor Receptor) signaling pathway.

In conclusion, our study showed that the expression of miR-148a was increased in GBM and the expression level was associated with the degree of malignancy of GBM. DLGAP1 is a novel target of miR-148a in GBM and mediates the effect of miR-148a on GBM cell migration and invasion. We used an iTRAQ-based quantitative proteomic approach combined with bioinformatics methods to uncover novel aspects of miR-148a-mediated pathways in GBM. Our findings provide a new mechanism for miR-148amediated GBM cell invasion and reveal previously unreported targets of miR-148a as well as novel aspects of miR148a-mediated regulatory networks in GBM. To further understand the role of miR-148a in GBM and to discover novel diagnostic and therapeutic strategies for GBM, there is an urgent need to establish transgenic mice that express miR-148a specifically in the brain, validate the oncogenicity and molecular regulatory network of miR-148a in vivo, and measure serum miR-148a expression in large cohorts of GBM patients using case-controlled methods.

\section{Materials and methods}

All experimental methods are described in more detail in Supplementary Information.

\section{Cells and tissue array}

Human GBM cell lines U87, U251, BT325, and T98G were obtained from Cobioer Biosciences (Nanjing, China) and a nonmaligant glial cell line, human astrocyte HA1800, was from Jennio Biotech (Guangzhou, China). The cell lines were cultured according to recommendations of the suppliers, in a humidified atmosphere of $5 \% \mathrm{CO}_{2}$ at $37^{\circ} \mathrm{C}$. All GBM cell lines were re-authenticated by STR (Short Tandem Repeat) profiling analysis. A tissue microarray, HBraGlio060PG-01, was obtained from Ribo Biotech (Guangzhou, China). All specimens of the tissue microarray were histologically confirmed, including 3 human normal brain tissues and 54 gliomas. The patients did not receive local or systemic radiotherapy and chemotherapy before surgery.

\section{Preparation of lentiviruses and establishment of pre-miR-148a and anti-miR-148a-stable expressing cells}

The miR-148a gene sequence and the miR-148a inhibitor oligonucleotides were chemically synthesized and inserted into the lentiviral vectors. The U251, U87, BT325, and T98G cells were used to establish pre-miR-148a and antimiR-148a-stable expressing cells by lentivirus methods.

\section{Rescue experiments}

To investigate whether DLGAP1 mediates the role of cell migration, invasion, and EMT in GBM, we transfected the shRNA-DLGAP1 virus into U87 and U251 GBM cells, and then evaluated the effect of DLGAP1 on cell migration and 
EMT. To determine whether the effects of miR-148a were mediated by DLGAP1, U87 cells stably expressing antimiR-148a were transfected with short hairpin RNA (shRNA) against DLGAP1, and cell migration, invasion, and EMT markers were measured as described above. All the experiments were performed in triplicate.

\section{Animal experiments}

Twenty-eight 4- to 6-week-old, 18-22 g, athymic male BALB/c nude mice were purchased from the Hunan SJA Animal Company (Hunan, China) and raised in specific pathogen-free conditions with $26-28^{\circ} \mathrm{C}$ and humidity of $40-60 \%$. The mice were divided equally into four groups using a random-number table (seven mice in each group) and implanted cells transfected with the pre-miR-148a-U87, pre-miR-con-U87, anti-miR-148a-U87, and anti-miR-conU87, respectively. The cells (about $1 \times 10^{6}$ ) were injected subcutaneously into the flank of the final volume of $100 \mu \mathrm{L}$. The length and the width of the visible tumors were measured 16 days after implantation by using Vemier calipers at regular intervals. Mice were killed when moribund or 28 days after initial injection of tumor cells, with the volume of the tumors to be calculated according to the formula $V=\left(\right.$ length $\times$ width $\left.^{2}\right) / 2$. The mice that died or did not grow tumors, or developed skin ulcers during the experiment were not included in the statistical analysis. The results were analyzed by SPSS 18.0 software. The measurement data were expressed as mean $\pm \mathrm{SD}$. Comparisons between groups were performed using repeated-measures analysis of variance (ANOVA) and a two-sided test was used, and the difference was statistically significant at $P \leq$ 0.05 . The animal experiments were approved by the Ethics Committee of Hunan Normal University and performed in accordance with the guidelines of Laboratory Animal Care (National Socitety for Medical Research).

\section{ITRAQ discovery experiments}

Sixty micrograms of protein from each sample was digested with $50 \mathrm{ng} / \mu \mathrm{L}$ trypsin and the resulting peptides were labeled with iTRAQ-8 Plex labeling (Applied Biosystems; Thermo Fisher Scientific, Inc., Waltham, MA, USA) according to the manufacturer's instructions; the peptide mixture from each sample was labeled as follows: the antimiR-con-U87 samples were labeled with iTRAQ 115 and 116, and the anti-miR-148a-U87 samples with iTRAQ 117 and 118 . The labeled samples were desalted by Ziptip, dried again by vacuum centrifugation, and then stored at $80^{\circ} \mathrm{C}$. The tryptic digest mixtures were separated by SCX (Strong Cation Exchange) chromatography using the Agilent 1100 HPL. Liquid chromatography MS/MS analysis was performed with a TripleTOF 5600 System (SCIEX, Concord,
ON, Canada) fitted with a Nanospray III source (AB Sciex, USA).

\section{Data analysis of iTRAQ experiments and bioinformatics analysis}

The MS/MS data were analyzed for protein identification and quantification using ProteinPilot Software v.4.5 (Sciex, Inc., USA). Using the integrated PSPEP tool in ProteinPilot software, the global error detection rate was estimated to be $1.0 \%$ by searching the uniprot-human protein database containing 20,210 protein sequences downloaded from the website (http://www.uniprot.org/uniprot/). All raw MS proteomics data and metadata have been deposited in iProx (http://www.iprox.org). The data set can be accessed from iProx with the identifier IPX0001370000. STarMir [30], combined with entropy and target gene structure features, was used to analyze the binding sites between miR-148aand miR-148a-regulated proteins discovered by iTRAQ. TarBase V8 was used to identify the confirmed targets and the novel targets of miR-148a [31]. A PPI network regulated by miR-148a was constructed using the human protein reference database (HPRD) [32]. The network characteristics were revealed through the degree of network graph, which was the number of edges connected to protein. The PPI network of miR-148a was generated using Cytoscape3.1 [51] and the degree of network was analysized by Network Analyzer App (https://techet.net/netanalyzer).

\section{Statistical analysis}

The data were statistically analyzed using Student's $t$-test, ANOVA, repeated-measures ANOVA, Kruskal-Wallis $H$ test, or $\chi^{2}$-test. The correlation analysis between the level of $m i R-148 a$ and DLGAP1 gene was performed using Spearman's correlation. Excel for the figures and SPSS 18.0 for statistical analyses were used. $P$-values were two-tailed and $P \leq 0.05$ was considered statistically significant.

Acknowledgements We are grateful to the members of the Basic Medical Laboratory Center of Hunan Normal University School of Medical for their technical support. We are grateful to members of the Animal Center of Hunan Normal University Medical School of Medicine for supporting animal experiments.

Funding This study was jointly supported by the National Natural Science Foundation of China (Grant Number 81472860) and the Construct Program of the Key Discipline of Basic Medicine in Hunan Normal University and Hunan Province.

Author contributions YL, WL, SZ, FZ, and XT conducted the experiments. WL, ZL, and $\mathrm{YZ}$ performed bioinformatic analysis. XZ, YL, SP, and Xiangni Peng performed statistical analysis. Xiaoning Peng, YL, WL, SP, XD, and ZL drafted the manuscript (writing, review, and/or revision of manuscript). TJR participated in the writing and revision of the whole manuscript. 


\section{Compliance with ethical standards}

Conflict of interest The authors declare that they have no conflict of interest.

Publisher's note: Springer Nature remains neutral with regard to jurisdictional claims in published maps and institutional affiliations.

Open Access This article is licensed under a Creative Commons Attribution 4.0 International License, which permits use, sharing, adaptation, distribution and reproduction in any medium or format, as long as you give appropriate credit to the original author(s) and the source, provide a link to the Creative Commons license, and indicate if changes were made. The images or other third party material in this article are included in the article's Creative Commons license, unless indicated otherwise in a credit line to the material. If material is not included in the article's Creative Commons license and your intended use is not permitted by statutory regulation or exceeds the permitted use, you will need to obtain permission directly from the copyright holder. To view a copy of this license, visit http://creativecommons. org/licenses/by/4.0/.

\section{References}

1. Ohgaki H. Epidemiology of brain tumors. Cancer Epidemiol. 2009;472:323-42.

2. Wen PY, Kesari S. Malignant gliomas in adults. New Engl J Med. 2008;359:492-507.

3. Ohgaki H, Kleihues P. Population-based studies on incidence, survival rates, and genetic alterations in astrocytic and oligodendroglial gliomas. J Neuropathol Exp Neurol. 2005;64:479-89.

4. Stupp R, Hegi ME, Mason WP, van den Bent MJ, Taphoorn MJ, et al. Effects of radiotherapy with concomitant and adjuvant temozolomide versus radiotherapy alone on survival in glioblastoma in a randomised phase III study: 5-year analysis of the EORTC-NCIC trial. Lancet Oncol. 2009;10:459-66.

5. Kim J, Zhang Y, Skalski M, Hayes J, Kefas B, Schiff D, et al. MicroRNA-148a is a prognostic oncomiR that targets MIG6 to regulate EGFR and apoptosis in glioblastoma. Cancer Res. 2014;74:1541-53.

6. McLendon R, Friedman A, Bigner D, Van Meir EG, Brat DJ, Olson $\mathrm{J}$, et al. Comprehensive genomic characterization defines human glioblastoma genes and core pathways. Nature. 2008;455:1061-8.

7. Xiong J, Bing Z, Su Y, Deng D, Peng X. An integrated mRNA and microRNA expression signature for glioblastoma multiforme prognosis. PLoS ONE. 2014;9:e98419.

8. Zheng B, Liang L, Wang C, Huang S, Cao X, Zha R, et al. MicroRNA-148a suppresses tumor cell invasion and metastasis by downregulating ROCK1 in gastric cancer. Clin Cancer Res. 2011;17:7574-83.

9. Zhang JP, Zeng C, Xu L, Gong J, Fang JH, Zhuang SM. MicroRNA-148a suppresses the epithelial-mesenchymal transition and metastasis of hepatoma cells by targeting Met/Snail signaling. Oncogene. 2014;33:4069-76.

10. Liffers ST, Munding JB, Vogt M, Kuhlmann JD, Verdoodt B, Nambiar S, et al. MicroRNA-148a is down-regulated in human pancreatic ductal adenocarcinomas and regulates cell survival by targeting CDC25B. Lab Investig. 2011;91:1472-9.

11. Yu J, Li Q, Xu Q, Liu L, Jiang B. MiR-148a inhibits angiogenesis by targeting ERBB3. J Biomed Res. 2011;25:170-7.

12. Wang H, Pan JQ, Luo L, Ning XJ, Ye ZP, Yu Z, et al. NF-kappa $\mathrm{B}$ induces miR-148a to sustain TGF-beta/Smad signaling activation in glioblastoma. Mol Cancer. 2015;14:2.
13. Roth P, Wischhusen J, Happold C, Chandran PA, Hofer S, et al. A specific miRNA signature in the peripheral blood of glioblastoma patients. J Neurochem. 2011;118:449-57.

14. Kim E, Naisbitt S, Hsueh YP, Rao A, Rothschild A, Craig AM, et al. GKAP, a novel synaptic protein that interacts with the guanylate kinase-like domain of the PSD-95/SAP90 family of channel clustering molecules. J Cell Biol. 1997;136:669-78.

15. Satoh K, Yanai H, Senda T, Kohu K, Nakamura T, Okumura N, et al. DAP-1, a novel protein that interacts with the guanylate kinase-like domains of hDLG and PSD-95. Genes Cells. 1997;2:415-24.

16. Manneville JB, Jehanno M, Etienne-Manneville S. Dlg1 binds GKAP to control dynein association with microtubules, centrosome positioning, and cell polarity. J Cell Biol. 2010;191:585-98.

17. Bilder D. Epithelial polarity and proliferation control: links from the Drosophila neoplastic tumor suppressors. Genes Dev. 2004;18:1909-25.

18. Zhan L, Rosenberg A, Bergami KC, Yu M, Xuan Z, Jaffe AB, et al. Deregulation of scribble promotes mammary tumorigenesis and reveals a role for cell polarity in carcinoma. Cell. 2008; 135:865-78.

19. Pearson HB, Perez-Mancera PA, Dow LE, Ryan A, Tennstedt $P$, et al. SCRIB expression is deregulated in human prostate cancer, and its deficiency in mice promotes prostate neoplasia. J Clin Investig. 2011;121:4257-67.

20. Li Y, Deng X, Zeng X, Peng X. The role of Mir-148a in cancer. J Cancer. 2016;7:1233-41.

21. Han CL, Chien CW, Chen WC, Chen YR, Wu CP, Li H, et al. A multiplexed quantitative strategy for membrane proteomics opportunities for mining therapeutic targets for autosomal dominant polycystic kidney disease. Mol Cell Proteomics. 2008;7:1983-97.

22. Lee JK, Joo KM, Lee J, Yoon Y, Nam DH. Targeting the epithelial to mesenchymal transition in glioblastoma: the emerging role of MET signaling. Oncotargets Ther. 2014;7:1933-44.

23. Huang DW, Sherman BT, Lempicki RA. Systematic and integrative analysis of large gene lists using DAVID bioinformatics resources. Nat Protoc. 2009;4:44-57.

24. Cavallaro U, Christofori G. Cell adhesion and signalling by cadherins and Ig-CAMs in cancer. Nat Rev Cancer. 2004;4:118-32.

25. Cui D, Sajan P, Shi J, Shen Y, Wang K, Deng X, et al. MiR-148a increases glioma cell migration and invasion by downregulating GADD45A in human gliomas with IDH1 R132H mutations. Oncotarget. 2017;8:25345-61.

26. Lee HK, Xiang C, Cazacu S, Finniss S, Kazimirsky G, Lemke N, et al. GRP78 is overexpressed in glioblastomas and regulates glioma cell growth and apoptosis. Neuro Oncol. 2008;10:236-43.

27. Jin SG, Dai Y, Li C, Fang X, Han HJ, Wang DX. MicroRNA-544 inhibits glioma proliferation, invasion and migration but induces cell apoptosis by targeting PARK7. Am J Transl Res. 2016;8:1826-37.

28. Sims JN, Graham B, Pacurari M, Leggett SS, Tchounwou PB, Ndebele K, et al. Di-ethylhexylphthalate (DEHP) modulates cell invasion, migration and anchorage independent growth through targeting S100P in LN-229 glioblastoma cells. Int J Environ Res Public Health. 2014;11:5006-19.

29. Hu CW, Tseng CW, Chien CW, Huang HC, Ku WC, Lee SJ, et al. Quantitative proteomics reveals diverse roles of miR-148a from gastric cancer progression to neurological development. J Proteome Res. 2013;12:3993-4004.

30. Rennie W, Kanoria S, Liu C, Mallick B, Long D, Wolenc A, et al. STarMirDB: a database of microRNA binding sites. RNA Biol. 2016;13:554-60.

31. Karagkouni D, Paraskevopoulou MD, Chatzopoulos S, Vlachos IS, Tastsoglou S, Kanellos I, et al. DIANA-TarBasev8: a decadelong collection of experimentally supported miRNA-gene interactions. Nucleic Acids Res. 2018;46:D239-45. 
32. Keshava Prasad TS, Goel R, Kandasamy K, Keerthikumar S, Kumar S, et al. Human Protein Reference Database-2009 update. Nucleic Acids Res. 2009;37:D767-72.

33. Kalluri R, Weinberg RA. The basics of epithelial-mesenchymal transition. J Clin Investig. 2009;119:1420-8.

34. Kalluri R, Neilson EG. Epithelial-mesenchymal transition and its implications for fibrosis. J Clin Investig. 2003;112:1776-84.

35. Mittal V. Epithelial mesenchymal transition in tumor metastasis. Annu Rev Pathol Mech Dis. 2018;13:395-412.

36. Polyak K, Weinberg RA. Transitions between epithelial and mesenchymal states: acquisition of malignant and stem cell traits. Nat Rev Cancer. 2009;9:265-73.

37. Tso CL, Shintaku P, Chen J, Liu Q, Liu J, Chen Z, et al. Primary glioblastomas express mesenchymal stem-like properties. Mol Cancer Res. 2006;4:607-19.

38. Elias MC, Tozer KR, Silber JR, Mikheeva S, Deng M, Morrison RS, et al. TWIST is expressed in human gliomas and promotes invasion. Neoplasia. 2005;7:824-37.

39. Qin Y, Capaldo C, Gumbiner BM, Macara IG. The mammalian Scribble polarity protein regulates epithelial cell adhesion and migration through E-cadherin. J Cell Biol. 2005;171:1061-71.

40. McCaffrey LM, Montalbano J, Mihai C, Macara IG. Loss of the Par3 polarity protein promotes breast tumorigenesis and metastasis. Cancer Cell. 2012;22:601-14.

41. Sánchez-Valle J, Tejero H, Ibáñez K, Portero JL, Krallinger M, Al-Shahrour F, et al. A molecular hypothesis to explain direct and inverse co-morbidities between Alzheimer's disease, glioblastoma and lung cancer. Sci Rep. 2017;7:4474.

42. Hsu CW, Juan HF, Huang HC. Characterization of microRNAregulated protein-protein interaction network. Proteomics. 2008;8:1975-9.
43. Bethke L, Sullivan K, Webb E, Murray A, Schoemaker M, Auvinen A, et al. The common D302H variant of CASP8 is associated with risk of glioma. Cancer Epidemiol Biomark Prev. 2008;17:987-9.

44. Skiriute D, Vaitkiene P, Saferis V, Asmoniene V, Skauminas K, Deltuva VP, et al. MGMT, GATA6, CD81, DR4, and CASP8 gene promoter methylation in glioblastoma. Bmc Cancer. 2012;12:218.

45. Freed E, Lacey KR, Huie P, Lyapina SA, Deshaies RJ, Stearns T, et al. Components of an SCF ubiquitin ligase localize to the centrosome and regulate the centrosome duplication cycle. Genes Dev. 1999; 13:2242-57.

46. Kipreos ET, Pagano M. The F-box protein family. Genome Biol. 2000;3002:1-7.

47. Piva R, Liu J, Chiarle R, Podda A, Pagano M, Inghirami G. In vivo interference with Skp1 function leads to genetic instability and neoplastic transformation. Mol Cell Biol 2002;22:8375-87.

48. Huang J, Peng X, Zhang K, Li C, Su B, Zhang Y, et al. Coexpression and significance of Dok2 and Ras $\mathrm{p} 21$ protein activator 1 in breast cancer. Oncol Lett. 2017;14:5386-92.

49. Chen YL, Huang WC, Yao HL, Chen PM, Lin PY, Feng FY, et al. Down-regulation of RASA1 is associated with poor prognosis in human hepatocellular carcinoma. Anticancer Res. 2017;37:781-5.

50. Hatanpaa KJ, Burma S, Zhao D, Habib AA. Epidermal growth factor receptor in glioma: signal transduction, neuropathology, imaging, and radioresistance. Neoplasia. 2010;12:675-84.

51. Smoot ME, Ono K, Ruscheinski J, Wang PL, Ideker T. Cytoscape 2.8: new features for data integration and network visualization. Bioinformatics. 2011;27:431-2. 